\title{
MAGNETO STATIC ANALYSIS OF ADHESION FORCE FOR THE WALL CLIMBING ROBOT
}

\author{
SHYAMAL C. MONDAL and ZOLJARGAL FALLOWS \\ London South Bank University \\ School of Engineering \\ Mechanical and Design \\ 103 Borough Road, London SE1 OAA
}

\begin{abstract}
This paper describes the analysis and results of the magneto static adhesion force which required for the climbing robot to climb the high walls when additional loads are need to be carried such as scanning devices for the non-destructive testing inspection of nuclear plants or high rise wind towers. Magneto-static simulations and experiments have been carried out for the investigation of the parameters of magnetic adhesion system and the static analysis of a climbing robot is performed with regard to anti-slipping forces and anti-overturning moments. The results from both simulations and experiments show that the adhesion mechanism with just two N52 magnets has enough adhesion capacity to carry a payload exceeding $300 \mathrm{~N}$. The prototype wheeled locomotion robot is built and magnetic adhesion mechanism has been mounted on the belly of a robot. The simulation and experimental results are compared. The experiment has been carried out in the robotic laboratory on the vertical surface and the result from the prototype show that the adhesion mechanism provides enough adhesion force for the prototype designed robot.
\end{abstract}

\section{Introduction}

A Permanent magnetic contact or non-contact with the surface system design for wall climbing robot is common. Basically, the robot consist of framework, two servo motors and tracked wheels. Each track has two sprockets, roller chain and evenly spaced permanent magnetic units. Two independent motors are used to drive the tracks on each side of the robot allowing it have good manoeuvrability and rotate about its own axis. Furthermore, when the robot in motion there are always set number of magnetic units in contact with the surface resulting in more reliable attachment to surface. The magnetic wheel mechanism can move on vertical surface and good turning capability. However, the adsorption area is limited and there is a fixed air-gap, thus the energy ratio limited to minimal [ 14 $6]$.

Symmetrically centralised magnetic wheel unit for wall climbing robots have been proposed by Kang Liu and the others. The unit consists of wheel, permanent magnetic blocks and connecting parts. The unit has a fixed number of permanent magnets symmetrically arranged around the wheel and there is always a certain air-gap between the magnet blocks and the surface. The characteristics of the design provides stable adsorption for between the magnetic blocks and the surface with different curvature radius. The tracked type mechanism has large adsorption area and great adhesion force due to the small gap between the track magnets and the ferromagnetic surface when active. Even though, it can rotate about its axel; it is not really flexible as the wheeled type thus making it harder to turn [2 5].

Minghui $\mathrm{Wu}$ and the others have proposed a non-contact adjustable permanent magnetic adhesion mechanism for a wall climbing welding robot $[3$ $6]$. The magnets are mounted under the mobile a platform and there is an air-gap between the permanent magnets and the ferromagnetic surface. The layout of the magnets offer enough adsorption area to generate enough adhesion force for the robot to carry heavy payload and pass obstacles. The non-contact adjustable permanent magnetic adhesion mechanism can overcome these disadvantages by utilising a large absorption area with certain air-gap and lifting mechanism for passing obstacles. 
For turbine inspection robot, the obstacles does not have to be considered but a large adsorption area and high adhesion force is essential as it is required to carry heavy payload. Thus, the non-contact adjustable permanent magnetic adhesion mechanism has most compatible system principle for the robot. However, the weight of this design is the main concern; mainly due to its lifting mechanism. In conclusion, embedded magnetic adhesion mechanism can be implemented for in this project and subsequent simulations or experiments can be carried out in order to investigate the permanent magnetic adhesion force. Furthermore, lifting mechanism is not needed as there is no obstacle for the robot to tackle.

This paper investigates an analysis of forces and moments required in order to achieve the minimal sliding force and overturning moment that oppose adhesion forces when a robot is attached to a vertical surface.

\section{Static analysis of a climbing robot}

When the robot stays static on a vertical surface, the forces acting on it are gravitational force, the adhesion force generated by the permanent magnetic unit, the supporting force of the surface and the frictional force between the drive member and the surface it moves on, which are denoted as $G, F_{m}, N$ and $F_{f}$, respectively. The adhesion force is the most critical part for the climbing robot as it keeps the robot attached to the surface.

\subsection{Sliding and Slipping analysis of a climbing robot}

For a climbing robot, it is ideal to avoid sliding and slipping during transition or moving on an inclined surfaces. To understand the forces, we must consider the static forces that acting on a robot, where a robot is resting on a inclined surface with angle, $\theta$, as shown in Fig. 1 .

This allows us to determine the minimum adhesion force for a robot to keep stay static on a vertical surface without slipping or slipping. Furthermore, by tapering the angle of the wall, the friction force will have bigger impact, thus the adhesion force it needs to keep stay on the surfaces reduced proportionally to the angle.
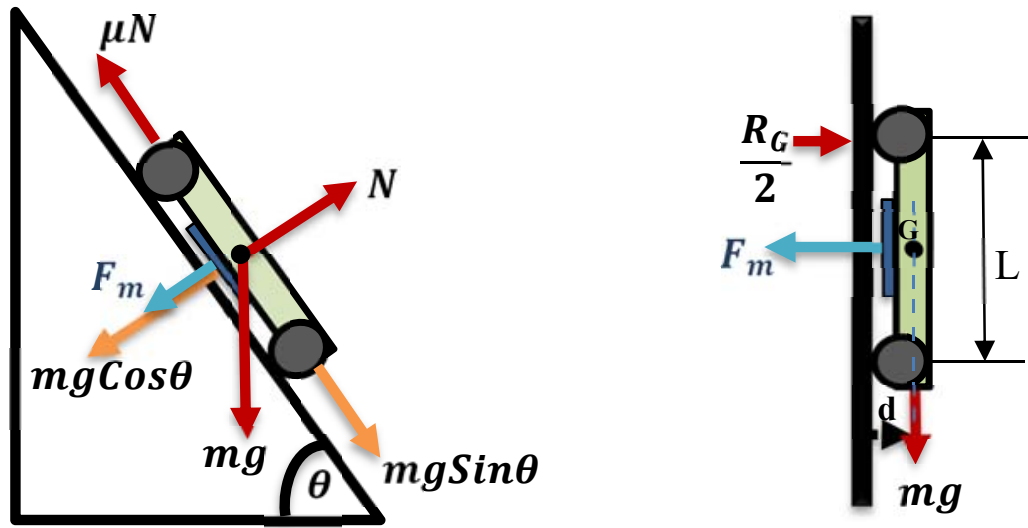

Fig.1 -Robot on inclined surface 
For a robot stay under the condition of static equilibrium, it must obey:

$$
\begin{aligned}
& \uparrow^{+} \Sigma \mathrm{F}_{\mathrm{y}}=0 \quad \text { /l }-\mathrm{mg} \operatorname{Cos} \theta-\mathrm{F}_{\mathrm{m}}+\mathrm{N}=0 ; \mathrm{N}=\mathrm{mg} \operatorname{Cos} \theta+\mathrm{F}_{\mathrm{m}} \\
& \rightarrow^{+} \Sigma \mathrm{F}_{\mathrm{x}}=0 \quad \geqslant \mathrm{mgSin} \theta-\mu \mathrm{N}=0 ; \mathrm{N}=\frac{\mathrm{mgSin} \theta}{\mu} \text { and } \mathrm{mgC \operatorname {Cos }} \theta+\mathrm{F}_{\mathrm{m}}=
\end{aligned}
$$

Thus, for the robot to avoid slipping: $\quad F_{m}>\frac{m g \operatorname{Sin} \theta}{\mu}-m g \operatorname{Cos} \theta$

In the case of the robot to climb on a pure vertical surface where the angle, $\theta=90$, the magnetic adhesion force becomes: $\mathbf{F}_{\mathbf{m}}>\frac{\mathbf{m g}}{\boldsymbol{\mu}}$

The calculations suggest that the minimum magnetic adhesion force that needed to avoid slipping must be greater than $\mathrm{mg} / \mu$. The Fig. 2 states that the performance of the robot can be improved by decreasing the weight of the robot or increasing the friction coefficient between the drive member mechanism and the surface it moves on. Furthermore, high friction is desirable for a wheeled climbing robot as the motion can only be provided from friction. However, the friction coefficient might change depending on the environment and aerodynamic resistance, where weather condition has major impact on the performance of the robot

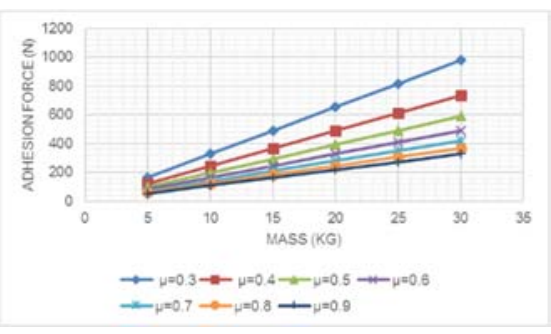

Fig. 2 - Minimum adhesion at different friction coefficient, $\mu$

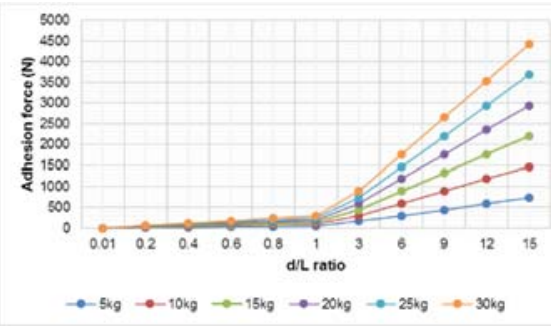

Fig.3 - Effect of d/L on Adhesion force

\subsection{Overturning analysis of a climbing robot}

Avoidance of overturning is a very subject for climbing robot, which subsequently keeps it attached to a vertical surface during operation. The sum of the moments at centre point $\mathrm{G}$ is $\mathrm{M}_{\mathrm{g}}, \mathrm{R}_{\mathrm{G}}$ - reaction force. At equilibrium, it obeys:

$$
\Sigma \mathrm{M}_{\mathrm{G}}=0 \quad ; \quad \mathrm{mg} \times \mathrm{d}+\frac{\mathrm{R}_{\mathrm{G}}}{2} \times \mathrm{L}=0 \quad \text { and } \mathrm{R}_{\mathrm{G}}=-\frac{2 \mathrm{mgd}}{\mathrm{L}}
$$

For a wheeled climbing robot, magnetic adhesion force is shared equally among the wheels. Thus, magnetic adhesion force can be considered: $F_{m}=\frac{R_{G}}{4}$ or $4 \mathrm{~F}_{\mathrm{m}}=\mathrm{R}_{\mathrm{G}}$

For the robot to avoid overturning, the magnetic adhesion force must satisfy following equation: $\mathbf{F}_{\mathbf{m}}>\frac{\mathbf{m g d}}{2 \mathbf{L}}$

The calculations state that by decreasing the ratio $\mathrm{d} / \mathrm{L}$, (where L- distance between front and rear wheel, $\mathrm{d}-$ the distance between the surface and centre of 
gravity line) the overturning of the robot can be avoided. Which means that the centre of the gravity of robot should be as close as possible to the surface and the distance between the front and rear wheels, where taking L large as possible. However, this this increase the overall weight of the robot, subsequently the adhesion force that needed to avoid overturning also increases, therefore it should be optimised.

In conclusion, the following criteria determines the minimum magnetic adhesion for a robot to stay statically equilibrium on a vertical, without slipping and overturning: $\quad \mathbf{F}_{\mathbf{m}}>\left\{\frac{\mathbf{m g}}{\boldsymbol{\mu}} ; \frac{\mathbf{m g d}}{\mathbf{2 L}}\right\}$

The calculated results from the static analysis determine that overturning can be avoided as long as slipping of the robot overcome with sufficient friction and adhesion force. Moreover, the friction force generated should be greater than the robot weight in order to any slippage, thus a higher friction coefficient will improve the stability of the robot.

\section{Magneto-static simulation}

Permanent magnetic adhesion system mainly depends upon the magnetic field distribution and the structural size of the device, which has been optimized through the Finite Element Analysis (FEA). For the simulation, rear earth Neodymium magnet (N52) is used. The characteristics of the magnet used for the simulation is shown in Table 1.

\subsection{Distance between magnets on Adhesion Force}

When two magnets set on a yoke, the distance between magnets has effect on the magnetic flux density which would affect the total force. For this simulation, two pieces N52 magnets with $10 \mathrm{~mm}$ thickness, $25 \mathrm{~mm}$ length and $50 \mathrm{~mm}$ width, are placed on iron bar (flat) with $10 \mathrm{~mm}$ thickness, and steel plate with $5 \mathrm{~mm}$ thickness. The air-gap between the magnets and the steel plate is kept constant at $5 \mathrm{~mm}$ for this simulation. By varying the distance between the magnets, magnetic field and flux density were observed.
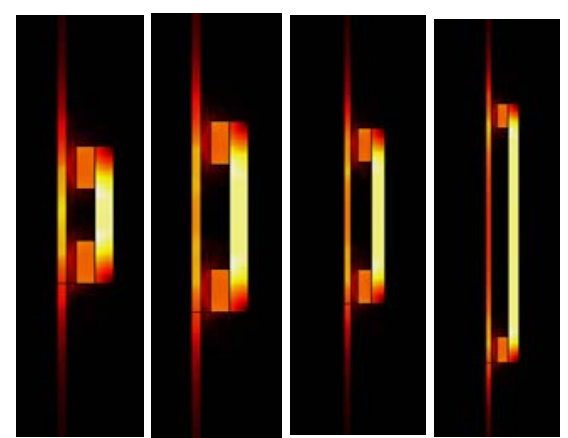

Fig.4 - Magnetic flux density at distance of $30 \mathrm{~mm}$, $60 \mathrm{~mm}, 80 \mathrm{~mm}$ and $200 \mathrm{~mm}$ between two magnets
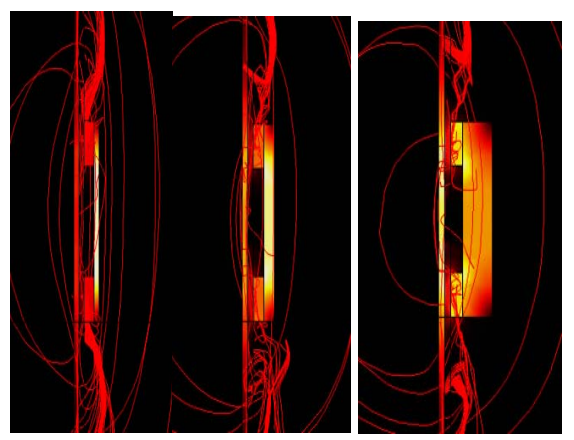

Fig.6 - Magnetic field and flux density with yoke thickness of $5 \mathrm{~mm}, 10 \mathrm{~mm}$ and $25 \mathrm{~mm}$

Simulation results states that the magnetic flux density in the steel plate increases with the distance between magnets but it starts to decrease significantly after the distance between the magnets gets bigger than 60 to $80 \mathrm{~mm}$. The hotspot shows that when the distance between the magnets is kept at $200 \mathrm{~mm}$, the magnetic flux concentration become much less, compared to $60 \mathrm{~mm}$, which means that the flux lines take much longer to reach one magnet to other (north to south), as shown in Fig.4. 


\subsection{Thickness of Yoke on Adhesion force}

Previuos simulations for distance between magnerts show that the most optimum distance between the magnets is $60 \mathrm{~mm}$ for $10 \mathrm{~mm}$ thick yoke. To observe the effect of the thickness of the yoke on adhesion force, other parameter kept same as first simulation. The magnetic flux concentration increases with thickness of yoke as well as the magnetic flux leackages is been reduced respectively, as shown in fig.6. Therefore, increase in thickness of yoke induces higher adhesion force. However, the magnetic flux concentration in steel plate is significcally resduced and the adhesion force starts to fall when yoke thickness bigger than $25 \mathrm{~mm}$, as shown in Fig.7. Furtheremore, By varying the thickness of the yoke shows that the maximum adhesion force is induced when thickness of yoke between 10 to $25 \mathrm{~mm}$. However, thicker yoke is not ideal for a climbing robot as it adhesion force to wieght ratio would decrease significantly, which is not diserable. Therefore, most optimum yoke thickness is most likely $10 \mathrm{~mm}$.

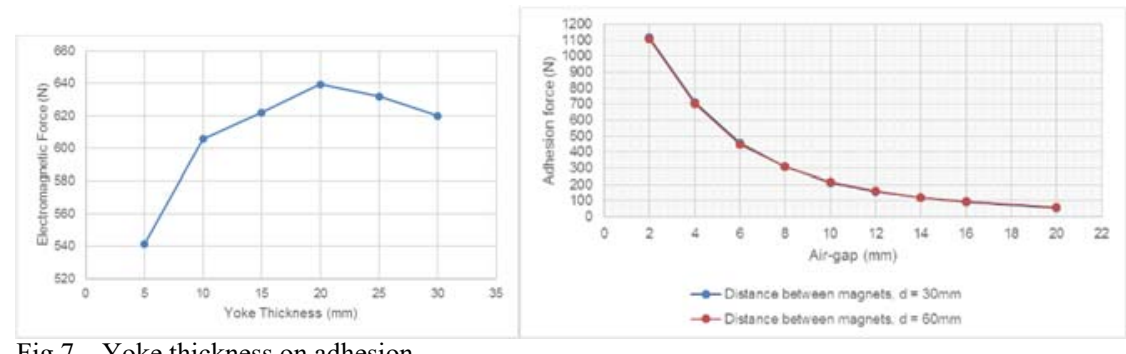

Fig.7 - Yoke thickness on adhesion Force

Fig. 8 - Air-gap between member of magnets and surface of steel plate on Adhesion Force

\subsection{Air-gap on Adhesion force}

To understand the effect of air-gap between the member of magnets and the surface of steel plate on the adhesion, the simulations were carried out accordingly.

Fig. 8 states that the adhesion force falls exponentially with increase in airgap, so in order to get high adhesion force air-gap has to small as possible. Even though, a small air-gap is desirable, the design criteria limits air-gap for climbing robot. Moreover, the magnetic flux concentrations in steel plate are almost identical when air-gap is at 2 to $5 \mathrm{~mm}$, for both cases. The optimum air-gap for the project is at $2 \mathrm{~mm}$, where magnetic flux leakage slightly more than $3 \mathrm{~mm}$ air-gap and with similar magnetic flux concentration.

Furthermore, the comparison of two different distances between the magnets are shown in Fig.8. The yellow bar represents the adhesion forces for $30 \mathrm{~mm}$ and the blue bar is for $60 \mathrm{~mm}$. When air-gap gets smaller than $8 \mathrm{~mm}$, adhesion force for $\mathrm{d}=30 \mathrm{~mm}$ is slightly higher than $\mathrm{d}=60 \mathrm{~mm}$ and slightly less when the air-gap gets bigger than $8 \mathrm{~mm}$. However, the behaviour of the both curves are almost identical. On the other hand, weight to adhesion force ratio of $60 \mathrm{~mm}$ is smaller than $30 \mathrm{~mm}$, which is due to the increase in yoke size for bigger $\mathrm{d}$.

This results are supported by the experiment. As expected, the Fig. 10 states the adhesion force drops exponentially with increase in air gap. However, adhesion force drops significantly at 2 to $8 \mathrm{~mm}$ of air-gap. On the other hand, the rate of drop in adhesion force much smaller at 8 to $10 \mathrm{~mm}$. This is mainly due to the magnetic flux concentration in the steel plate is reduced significantly for which flux leakage much greater at this point. 


\section{Magneto-static experimental setup}

The experiment was carried out to validate the simulation results. In this experiment, two weighing scales are used to measure the adhesion force that generated from the magnets at different parameters. In this experiment, only 2 different magnet arrangements are explored. The picture shown in Fig. 9 was taken during the experiment, to demonstrate the experiment approach.

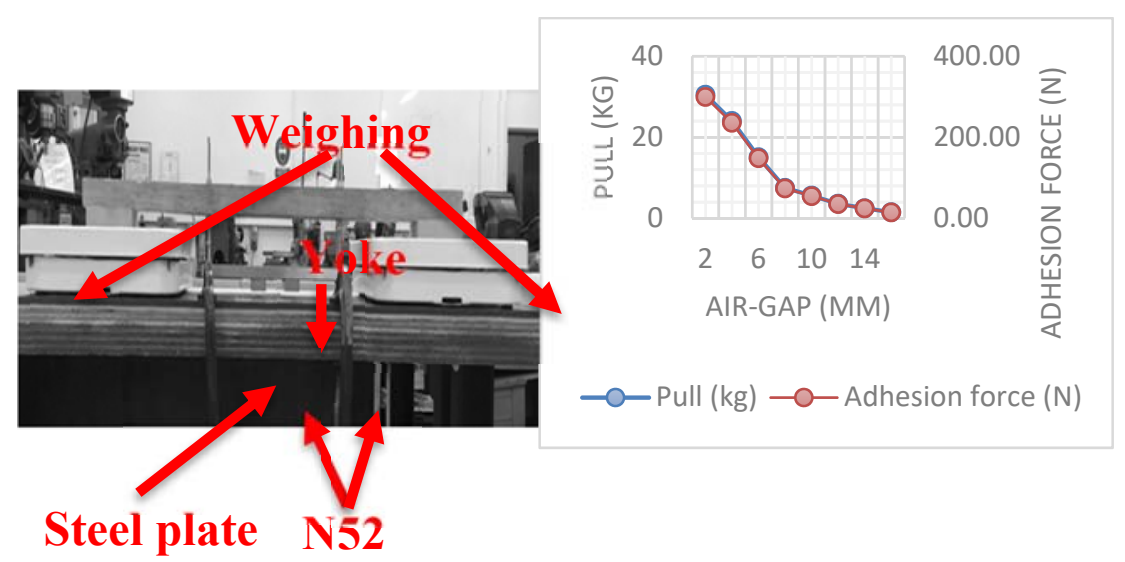

Fig. 9 -Experimental set-up

Fig.10 - Effect of air-gap on adhesion force

\subsection{Prototype design and implementation}

The prototype robot was designed based on the simulations results and calculations that has been carried out. The hardware of the robot consists of two different types of parts, electronic and mechanical. The mechanical components are consists of four $65 \mathrm{~mm}$ rubber wheels, upper and lower platforms, four DC motors and magnetic adhesion device. Upper platform is implemented to accommodate the electronic components, which offset from the lower platform by $30 \mathrm{~mm}$, in order to protect the electronic components from magnetic flux leakages from which induced from the magnetic adhesion device. Moreover, the upper and lower platforms are made out of $6 \mathrm{~mm}$ and $3 \mathrm{~mm}$ acrylic, respectively in order to minimise the weight of the robot.

Four wheel drive is implemented for better friction force from the wheels. Rubber wheels with diameter of $65 \mathrm{~mm}$ is chosen for the prototype to increase the traction between the drive member and the surface which it climbs. DC motors has torque of $0.49 \mathrm{Nm}$ at maximum rotational speed of $37 \mathrm{rpm}$. Total weight of the prototype is $1.54 \mathrm{~kg}(15.1 \mathrm{~N})$. Static analysis suggests that the overturning and slipping can be avoided as long as the adhesion force is sufficient enough to keep the prototype attached to a wall. According to the experimental results, the airgap between the adhesion mechanism and ferromagnetic surface must be less than $15 \mathrm{~mm}$, in order to provide keep the prototype robot attached to a vertical wall. On the other hand, the simulation analysis showing that the air-gap can be increased to as high as $20 \mathrm{~mm}$. However, when the robot attached to a wall, the payload increases significantly due to the adhesion force.

For testing the climbing capability of the prototype robot, it was carried out on a vertical steel wall in robotic lab as shown in Fig. 11 (a \& b). The robot was placed on a vertical surface with $10 \mathrm{~mm}$ of air-gap to check its adhesive force. According to the experiment results, it is assumed to be the minimum air-gap the motors could operate at although the simulation results suggested a slightly bigger air-gap in order to move the robot on the vertical surface. Furthermore, the torque required from each wheel has to be bigger than $1.72 \mathrm{Nm}$ at $10 \mathrm{~mm}$ air-gap for 
simulations, however it is much bigger than the maximum torque of the chosen motor.

Results showed, adhesive force was sufficient enough to keep the robot attached to the wall and produced a good friction at $10 \mathrm{~mm}$ air-gap. However, the robot was unable to climb up the wall when an additional $5 \mathrm{~N}$ payload was added and started to make whizzing noise, which meant that the chosen motors were incapable of carrying that payload and any further increase in speed beyond 60 percent was too risky as it could cause permanent damage to the motors due high current from the power supplier. On the other hand, moving down and stopping was demonstrated by the robot with no difficulty. Thus, the correlation between the technical results and practical work show that the main issue was mostly with chosen motor.
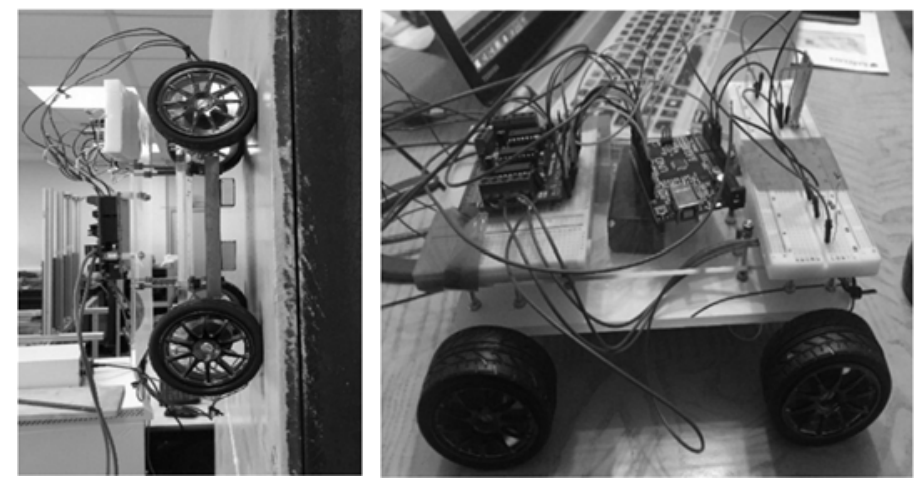

Fig. 11 - Prototype climbing robot : (a) Testing on the vertical surface hardware

\section{5}

\section{Conclusion}

A prototype wall climbing robot with magnetic adhesion mechanism is constructed to demonstrate the theory and analysed results for a wall vertical surface. Based on the investigation carried out on the working environment, a permanent magnetic adhesion mechanism was used for this application. However, there was one main issue with the robot as the torque required for the robot to climb is much higher than anticipated, for which higher torque motors were needed. On the hand, the robot seem have no difficulty of scaling down and stopping; thus, the overall performance of the robot was achieved and was able to induce enough adhesive force at $10 \mathrm{~mm}$ air-gap.

The experimental results states that the adhesion mechanism has a maximum payload capacity of $75 \mathrm{~N}$ at $10 \mathrm{~mm}$ air-gap. Furthermore, both simulation and experiment results show that adhesion force can be increased by introducing more magnets into the adhesion mechanism, which subsequently will increase the absorption area. In terms of magnetic adhesion, further research has been carried out in order to investigate the magnetic flux density in the steel plate with different thicknesses and carbon contents, and observed its effects on adhesion force. In extension, static and dynamic analysis of a climbing robot will need to be investigated further with respect to its change in orientation of the centre of mass, as well as real life testing.

There are some improvements that has to be made in order to transform the robot to real industrial use. A differential drive can be implement by introducing a tracked locomotion to the robot for better manoeuvrability and traction and an embedded pressure sensor can be implemented into the electrical system in order to monitor the payload during real life operation, which is essential as the payload increases greatly due to aerodynamic resistance. 


\section{References}

1. Anne Jungert "Damage Detection in Wind Turbine Blades using two Different Acoustic Techniques", The e-Journal of Nondestructive Testing (December, 2008)

2. Raju D.Dethe ,Dr. S.B. Jaju, "Developments in Wall Climbing Robots: A Review", International Journal of Engineering Research and General Science Volume 2, Issue 3, (April-May 2014)

3. Joseph L. Jones, Anita M. Flynn, Mechanics, Mobile Robots, Inspiration to Implementation, p139,

4. Weimin Shen, Jason Gu, "Permanent Magnetic System Design for the Wall-climbing Robot", Proceedings of the IEEE International Conference on Mechatronics \& Automation Niagara Falls, Canada, (July 2005)

5. Kang Liu, Wenzeng Zhang, "Symmetrically centralized magnetic-wheel unit for wall-climbing robots", Proceedings of the $13^{\text {th }}$ IASTED International Conference ROBOTICS AND APPLICATIONS, (August 2931, 2007)

6. Minghui Wu, Gen Pan, Tao Zhang, Shanben Chen, Fu Zhuang, Zhao Yanzheng, "Design and Optimal Research of a Non-Contact Adjustable Magnetic Adhesion Mechanism for a Wall-Climbing Welding Robot", International Journal of Advanced Robotic Systems, (October, 2012)

7. MD Omar faruq Howlader, (IJACSA) International Journal of Advanced Computer Science and Applications, Vol. 6, No. 8, 2015 